delivering safe care). We integrated a middle range psychological theory with our findings to recommend a focus for training nurses in streaming and service improvements. We recommend a collaborative approach to service development, guidance and training (including input from emergency department clinicians, primary care clinicians) and a range of training strategies that are suitable for less experienced junior nurses and more experienced senior nurses and nurse practitioners.

\section{EXTERNAL VALIDATION OF THE DUTCH PREDICTION MODEL FOR PREHOSPITAL TRIAGE OF TRAUMA PATIENTS IN SOUTH WEST REGION OF ENGLAND, UNITED KINGDOM}

${ }^{1}$ Thomas Shanahan, ${ }^{2}$ Carl Marincowitz, ${ }^{2}$ Gordon Fuller, ${ }^{3}$ Trevor Sheldon, ${ }^{4}$ Fionn Quilty, ${ }^{2}$ Emily Turton. 'Manchester Royal Infirmary; ${ }^{2}$ University of Sheffield; ${ }^{3}$ Barts and the London School of Medicine and Dentistry; ${ }^{4}$ Hull York Medical School

\subsection{6/emj-2020-rcemabstracts. 24}

Aims/Objectives/Background This is the first external validation of a European empirically derived prediction model for identifying major trauma in an unselected group of injured patients transported by ambulance in the United Kingdom.

Methods/Design This study was an external validation of a Dutch prediction model for identifying major trauma using a retrospective cohort of injured patients who ambulance crews transported to hospitals in the South West region of England. Major trauma was defined as Injury Severity Score (ISS) $>15$.

Participants were patients $\geq 16$ years with a suspected injury and transported by ambulance from February 1, 2017 to February 1,2018 . This study had a census sample of cases available to us over a one year period.

We tested the accuracy of the prediction model in terms of discrimination, calibration, clinical usefulness, sensitivity and specificity and under- and over triage rates compared to existing trauma triage practices in the South West region.

Results/Conclusions A total of 68698 adult patients were included in the final external validation cohort. The median age of patients was 72 (i.q.r. 46-84); 55.5\% were female; and $524(0.8 \%)$ had an ISS $>15$. In comparison the Dutch cohort was younger (45 years), more were male $(58.3 \%)$ and more patients had an ISS $>15$. (8.8\%) The model achieved good discrimination with a C-Statistic $0.75 \quad(95 \%$ CI, $0.73-0.78)$. At a maximal specificity of $50 \%$ the model resulted in a sensitivity of $86 \%$. The model improved undertriage rates at the expense of increased overtriage rates compared with routine trauma triage methods in the South West of England.

The Dutch prediction model for identifying major trauma can lower the undertriage rate to $17 \%$, however it would increase the overtriage rate to $50 \%$ in this UK cohort. Further research is needed to determine whether the model can be practically implemented by paramedics and is cost-effective.

\section{KILLED IN ACTION (KIA): AN ANALYSIS OF MILITARY PERSONNEL WHO DIED OF THEIR INJURIES BEFORE REACHING A DEFINITIVE MEDICAL TREATMENT FACILITY IN AFGHANISTAN (2004-2014)}

${ }^{1}$ Stacey Webster, ${ }^{2}$ Ed Barnard, ${ }^{2}$ Jason Smith, ${ }^{3}$ Max Marsden, ${ }^{2}$ Chris Wright. 'Derriford Hospital; ${ }^{2}$ ADMEM; ${ }^{3}$ ADMSAT

10.1136/emj-2020-rcemabstracts. 25
Aims/Objectives/Background Most fatalities from trauma, in civilian and military settings, die before reaching a hospital. However, no previous studies have comprehensively examined this phase of care. The aim of this study was to define the time interval between injury and death in UK military personnel who died pre-hospital from enemy action (Killed in Action, KIA).

Methods/Design The UK Joint Trauma Theatre Registry (JTTR) was used to identify all UK military personnel who died in Afghanistan (2004-2014). Through novel linkage of medical and tactical databases, an accurate timeline of events was obtained. Cause of death was determined from post mortem review. The primary objective was to report time between injury and death. Secondary objectives: mortality at key timepoints, the temporal lethality of different anatomical injuries, and trends in the case fatality rate (CFR, defined as deaths/ injuries $\mathrm{x} 100)$. Data are reported as $\mathrm{n}(\%)$, and median [interquartile range]. Proportions compared with a Fisher's exact test, and survival was with a Gehan-Breslow-Wilcoxon test; level of significance was corrected by Bonferroni.

Results/Conclusions 2413 UK personnel were injured in Afghanistan from 2004-2014; 448 died, a CFR of 18.6\%. 390 $(87.1 \%)$ of total deaths (KIA + Killed Non-Enemy Action) were prehospital. Complete timeline data were available for $n=303$ (87.1\%) KIA - this cohort had a median injury severity score of 75.0 [55.5-75.0]. The median time between injury and death in KIA was 0.0 [0.0-21.8] minutes; 173 $(57.1 \%)$ died immediately, and by $10 \mathrm{~min}$ more than twothirds had died. Primary injury to the head had a significantly shorter time to death compared to the abdomen and lower extremity (both $p<0.01$ ). Significant improvement in survival over the decade was due to a reduction in pre-hospital CFR without an increase in in-hospital CFR.

Over two-thirds of KIA deaths occurred within $10 \mathrm{~min}$ of injury. Improvement in the CFR in Afghanistan was predominantly in the prehospital phase.

\section{STUDY OF SHORTNESS OF BREATH (SOB) POINT-OF- CARE BIOMARKER PANEL IN PATIENTS PRESENTING WITH SHORTNESS OF BREATH IN EMERGENCY DEPARTMENT}

Tanmay Kumar Jha. Max Super Speciality Hospital

\subsection{6/emj-2020-rcemabstracts.26}

Aim Study of shortness of breath (SOB) point-of-care biomarker panel in patients presenting with shortness of breath in Emergency Department.

Objective To study the sensitivity and specificity of SOB pointof-care biomarker panel in diagnosing Acute Coronary Syndrome (ACS), Heart Failure (HF) and Pulmonary Embolism (PE).

Background Shortness of breath (SOB) is one of the commonest symptoms of patients presenting to the emergency department (ED).

The differential diagnosis of SOB is very vast, knowing the frequency and severity potential of ACS, HF and PE should be considered.

Methods/Design 165 consecutive subjects 18 or more years old, presenting to the ED due to a primary complaint of SOB were included in this prospective study.

After detailed history and thorough physical examination, the blood samples of patients included in the study were 
analysed using a SOB point-of-care biomarker panel (troponin I, myoglobin, CK-MB, D-dimer, and BNP at the time when patient presented with SOB within 15 minutes of patient's visit to the ED. Thirty-day follow-up for HF, ACS and PE was performed.

The following cut offs were used for determining a positive biomarker:

1. myoglobin $>107 \mathrm{ng} / \mathrm{mL}$,

2. CK-MB $>4.3 \mathrm{ng} / \mathrm{mL}$,

3. troponin I $>0.40 \mathrm{ng} / \mathrm{mL}$,

4. D-dimer $>500 \mathrm{ng} / \mathrm{mL}$, and

5. BNP > $100 \mathrm{ng} / \mathrm{mL}$.

CTCA, CTPA and 2D Echo were taken as the Gold Standard for the diagnosis of ACS, HF and PE.

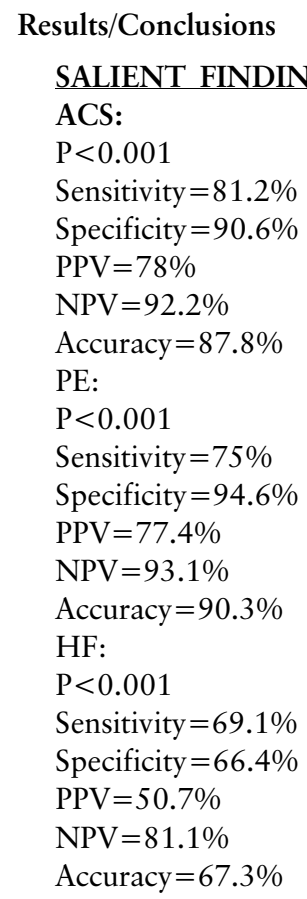

Conclusion We conclude that there is significant correlation between SOB point-of-care biomarker panel diagnosis and final diagnosis.

In patients presenting with $\mathrm{SOB}$, one of the three clinical conditions identified by the SOB point-of-care biomarker panel will lead to earlier diagnosis and initiation of specific management.

\section{POINT OF CARE LUNG ULTRASOUND IN PATIENT TRIAGE: INTEGRATION OF ULTRASOUND INTO A STREAMING PATHWAY FOR COVID-19}

Dominic Craver, Aminah Ahmad, Anna Colclough. Lewisham Hospital

10.1136/emj-2020-rcemabstracts.27

Aims/Objectives/Background Rapid risk stratification of patients is vital for Emergency Department (ED) streaming during the COVID-19 pandemic. Ideally, patients should be split into red (suspected/confirmed COVID-19) and green (non COVID-19) zones in order to minimise the risk of patient-to-patient and patient-to-staff transmission. A robust yet rapid streaming system combining clinician impression with point-of-care diagnostics is therefore necessary.
Point of care ultrasound (POCUS) findings in COVID-19 have been shown to correlate well with computed tomography (CT) findings, and it therefore has value as a front-door diagnostic tool. At University Hospital Lewisham (a district general hospital in south London), we recognised the value of early POCUS and its potential for use in patient streaming.

Methods/Design We developed a training programme, 'POCUS for COVID' and subsequently integrated POCUS into streaming of our ED patients. The training involved Zoom lectures, a face to face practical, a 10 scan sign off process followed by a final triggered assessment. Patient outcomes were reviewed in conjunction with their scan reports.

Results/Conclusions Currently, we have 21 ED junior doctors performing ultrasound scans independently, and all patients presenting to our department are scanned either in triage or in the ambulance. A combination of clinical judgement and scan findings are used to stream the patient to an appropriate area.

Service evaluation with analysis of audit data has found our streaming to be $94 \%$ sensitive and $79 \%$ specific as an indicator of COVID 19. Further analysis is ongoing.

Here we present both the structure of our training programme and our integrated streaming pathway along with preliminary analysis results.

\section{RCEM moderated}

\section{THE EFFECT OF AFFECT: WHAT WE TEACH OUR LEARNERS ABOUT THE ROLE OF PATIENT INDUCED EMOTION IN CLINICAL REASONING IN THE ED AND WHY?}

Susie Roy, Janet Skinner, Alan Jaap. NHS Lothian/University of Edinburgh

\subsection{6/emj-2020-rcemabstracts.28}

Aims/Objectives/Background Few empirical studies explore the contribution of non-clinical factors to perceptions of patient difficulty in EM. Fewer have investigated what students placed in EDs learn about 'difficult' patients or what, if anything, clinicians teach about the topic. We looked to address this. Considering these questions is imperative: patients perceived as frustrating report lower satisfaction with their clinical encounter, experience worse health outcomes and seem to be at risk of medical error secondary to faulty clinical reasoning.

Methods/Design With ethical approval, we undertook three interrelated, qualitative studies to conduct a case study of the undergraduate EM module delivered at Edinburgh University. In the first two, focus groups were used as the method of data collection; five clinician $(n=25)$ and four medical student $(n=21)$ groups were facilitated. In the third, semi-structured interviews with clinicians $(\mathrm{n}=12)$ were conducted. All groups/interviews were audio-recorded and transcribed. The data were analysed inductively using reflexive thematic analysis.

Results/Conclusions Frequent attendance, demands, pre-existing relationships and unrealistic expectations contributed to perceived patient difficulty. These were modified by personal and circumstantial factors. Although rarely told, students were aware who these 'difficult' patients were through observing behaviours. Critically, clinicians and students alike believed 